\title{
Microgrid reliability evaluation considering the intermittency effect of renewable energy sources
}

\author{
R. Ahshan ${ }^{\mathrm{a}}$, M. T. Iqbal ${ }^{\mathrm{b}}$, George K. I. Mann ${ }^{\mathrm{b}}$, John E. Quaicoe ${ }^{\mathrm{b}, *}$ \\ ${ }^{a}$ Sultan Qaboos University, Muscat,Al-Khod 123, Sultanate of Oman \\ ${ }^{b}$ Memorial University of Newfoundland, St. John's, A1B 3X5, Canada
}

\begin{abstract}
This paper presents the reliability evaluation of a microgrid system considering the intermittency effect of renewable energy sources such as wind in this study. One of the main objectives of constructing a microgrid system is to ensure reliable power supply to loads in the microgrid. In order to achieve this objective, it is essential to evaluate the reliability of power generation of the microgrid under various uncertainties. Because highly variable wind resources and different operating modes of the microgrid are the major factors to influence the generating capacity of the microgrid in this study. Reliability models of various sub-systems of a 3-MW wind generation system are developed. The sub-systems include wind turbine rotor, gearbox, generator, and interfacing power electronics system. The impact of stochastically varying wind speed to generate power by the wind turbine system is accounted in developing sub-systems reliability model. A Microgrid System Reliability (MSR) model is then developed by integrating the reliability models of wind turbine systems with hydro and storage units in the study microgrid system using the system reliability concept. A Monte Carlo simulation technique is utilized to implement the developed reliability models of wind generation and microgrid systems in Matlab environment. The investigation reveals that maximizing the use of wind generation systems and storage units increases the reliability of power generation of the proposed microgrid system in different operating modes.
\end{abstract}

Keywords: Reliability, distributed generation, microgrid, modeling and simulation

\section{Introduction}

Electricity market de-regulation, environmental concern, technology advancement and reduce dependency on fossil fuel are the main causes to integrate DG units into the distribution power network. Generally, DGs have diverse generation capacity, availability and primary energy sources. The increasing demand of adding and utilizing such diverse DGs into the distribution power system brought the concept of microgrid. Microgrid is a flexible combination of loads, DG units, storage systems (either centrally or with each generation individually), and associated power conditioning units operating as a single controllable system that provides power or both power and heat to loads [1]. Fig. 1 shows the generic architecture of a microgrid system.

One of the main objectives of having a microgrid system is to supply reliable power to loads in a microgrid domain. The achievement of such objective becomes critical when a microgrid system consists of renewable energy sources such as wind and/or solar. In the proposed microgrid system, stochastically varying wind causes unpredictable power output of the wind turbine system. In addition, such variations in wind speed propagates through all the sub-systems in the wind generation system. Therefore, sub-systems such as gearbox, generator and power electronics interfacing unit in a wind generation system are also the key factors for generating reliable power by the proposed microgrid system. Thus, it is important to develop the reliability model of the wind generation system including the models

\footnotetext{
* Manuscript received June 15, 2017; revised November 9, 2017.

Corresponding author. E-mail address: razzaqul@ squ.edu.om.

doi: $10.12720 /$ sgce.6.4.252-268
} 
of all the sub-systems. In addition, consideration of various operation modes of the microgrid system is important to develop microgrid system reliability model in order to ensure reliable power generation in those operating modes.

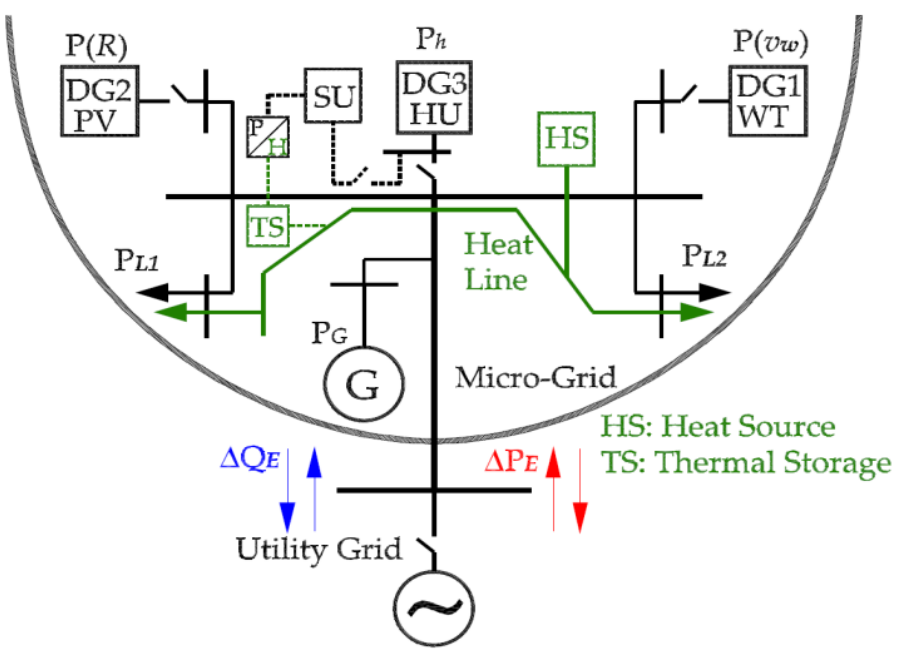

Fig. 1. A typical microgrid system.

Several researchers address the issues on microgrid architectures and their control systems development [1]-[13]. The operation, control, and performance characteristics of these microgrids are different because of the contribution of different diverse nature and sizes of distributed generations in the microgrid. Such distributed generations are fixed or variable speed wind turbines, solar panels, microturbines, various types of fuel cells, small hydro and storage depending upon the sites and resources available. Different control strategies such as load-frequency control, power sharing among parallel converters, central control based on load curve, active power control are developed for the microgrids presented in [1]-[13]. Reliability study of a microgrid system is presented in [14], where the concentration is given in power quality aspect based on the assumption that the microgrid system is a large virtual generator that has the ability to generate sufficient power for loads at various operating conditions. Reliability based co-ordination between wind and hydro system is investigated, which shows the adequacy benefits due to the coordination between them when an appropriate number of hydro units are engaged to follow the wind speed changes based on the wind power penetration [15]. Reliability and cost assessment of a solar-wind-fuel cell based microgrid system is investigated in [16]. A recent review study on reliability and economic evaluation of power system is presented in [17]. It is suggested that the reliability and economic evaluation of power systems with renewable energy sources needs to perform simultaneously. In [18], a new indicator for measuring reliability of a solar-wind microgrid system is presented. Reliability evaluation of distribution system that consists of wind-storagephotovoltaic system is presented in [19]. It shows the enhancement in reliability of the conventional distribution system using the renewable energy sources. In compare to microgrid architectures and control research, the investigation of reliability evaluation of micro-grid systems is few. Therefore, much attention is required to the reliability evaluation of a wind-hydro-storage based microgrid as it has significant potential in many places around the world.

Several researchers have studied reliability assessment of wind turbine generator in power system application [20]-[26]. The application of two-state and multi-state model for wind turbine systems are investigated in [20]-[22]. However, the stochastic variation and interactions of wind speed and thus time dependent wind power effects are avoided [27]. A Monte Carlo simulation based method is then used to evaluate wind generation system reliability in [15], [23]-[26]. All these past studies evaluate reliability of wind turbine system by determining the available power output using eq. 1, while the effect of other subsystems such as gearbox, generator, and interfacing power electronics has not been considered. 


$$
P_{o}= \begin{cases}0 & 0 \leq v_{w} \leq v_{c i w} \\ \left(A+B v_{w}+C v_{w}^{2}\right) P_{r} & v_{c i w} \leq v_{w} \leq v_{r w} \\ P_{r} & v_{r w} \leq v_{w} \leq v_{c o w} \\ 0 & v_{w} \geq v_{c o w}\end{cases}
$$

where, $P_{o}$ and $P_{r}$ are rotor output power and rated power of the wind turbine, respectively. $v_{c i w}, v_{r w}$, and $v_{\text {cow }}$ are cut-in, rated, and cut-out wind speed, respectively, and the parameters $A, B$, and $C$ are the functions of cut-in, rated and cut-out wind speed.

Moreover, these approaches determine available power only at the output of the WT rotor without considering the role of the other sub-systems. Reliability evaluation is carried out only for interfacing power electronics sub-system to compare performances of small $(1.5 \mathrm{~kW})$ wind generation system is presented in [28]. Furthermore, such reliability assessment of the interfacing power electronics subsystem is carried out for a single operating point such as at the rated wind speed condition. However, operating conditions of a wind generation system normally vary between cut-in to cut-out wind speed due to the stochastic behaviour of the wind speed. Hence, reliability evaluation of generating power by wind generation system is important to carry out considering the stochastic variation of wind speed as well as the impact of stochastic wind behaviour on different sub-systems in a wind generation system. Such considerations are important to achieve better reliability estimation to ensure reliable power supply by the microgrid system.

The reliability of power generation by a microgrid system consisting of wind generation, hydro generation, and storage unit is evaluated and presented. The case study microgrid system is located at Fermeuse, Newfoundland, Canada. The reliability model of the microgrid system is developed using the concept of reliability block diagram. The reliability modeling of the wind generation system is developed using wind speed data modeling. In addition, the reliability models of the sub-systems in the wind generation unit are developed based on the failure rate of the wind data using wind data modeling. Finally, the models are implemented using Monte Carlo simulation in Matlab environment. The results from this study indicate that:

a. The proposed microgrid system is able to provide reliable power to an isolated microgrid with a minimum number of wind power generation unit (only one) with a reliability of 0.94 .

b. However, maximizing the use of wind generation unit (as the number increases) improves the microgrid system reliability to provide reliable power to the isolated microgrid.

c. Due to the lack of sufficient wind, the integration of pumped hydro storage increases the microgrid system reliability to ensure reliable power supply to the isolated microgrid system.

\section{Micro-grid System Reliability}

The one-line diagram of the case study microgrid system shown in Fig. 2 consists of a HGU, a WPGS or a Wind Farm (WF), and two load areas represented as $\mathrm{P}_{\mathrm{L} 1}$ and $\mathrm{P}_{\mathrm{L} 2}$. HGU and WPGS are apart from each other by a TL1 (20.12) km transmission line.

Microgrid System Reliability (MSR) is a measurement of the system overall ability to produce and supply electrical power. Such measurement indicates the adequacy of power generation and supply by a microgrid system for a given combination of DG units in the system as well as the sub-systems contained in a DG unit. In order to evaluate reliability of the system shown in Fig. 2, the combination of DG units and the sub-systems contained in a DG unit can be presented by the concept of Reliability Block Diagram (RBD) [29]. Fig. 3 shows the RBD of the case study microgrid system. It is worth mentioning that the concentration in this study is given to evaluate the reliability of generating and supplying power by the microgrid system, hence only DG units are considered. The simplified $\mathrm{RBD}$ of the microgrid system is presented in Fig. 4, where all DG units are connected in parallel. 
However, the RBD of the microgrid system at different operational modes is shown in Fig. 5.

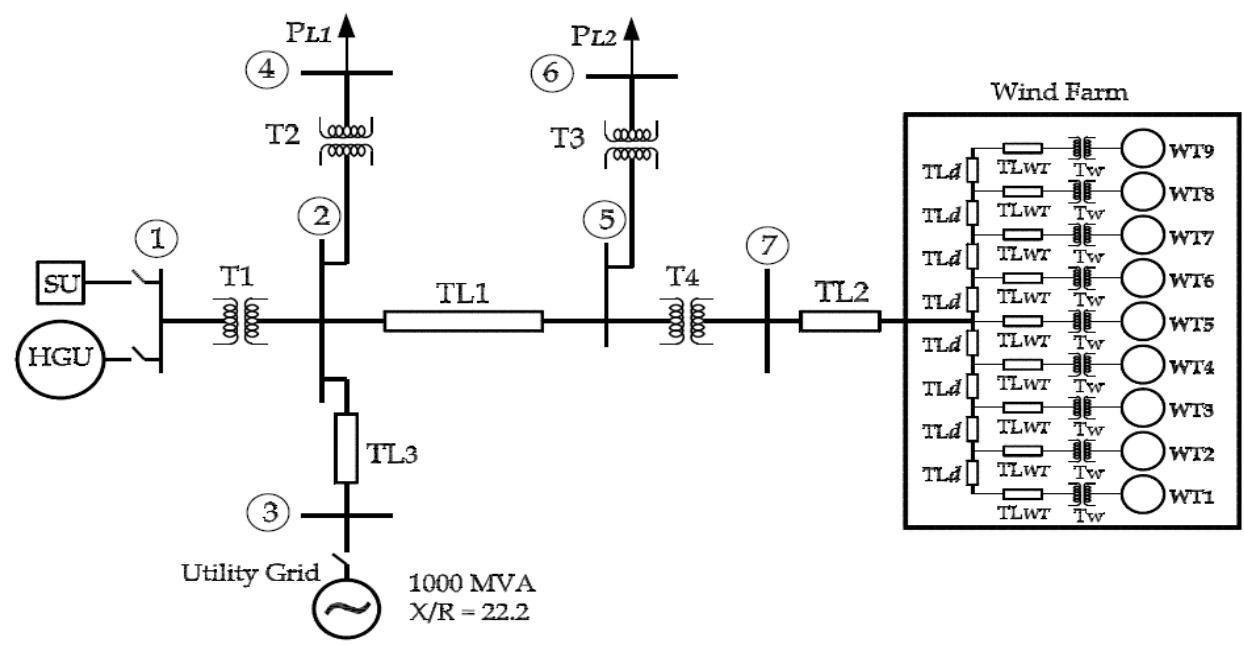

Fig. 2. The single-line diagram of a microgrid system at Fermeuse, Newfoundland, Canada.

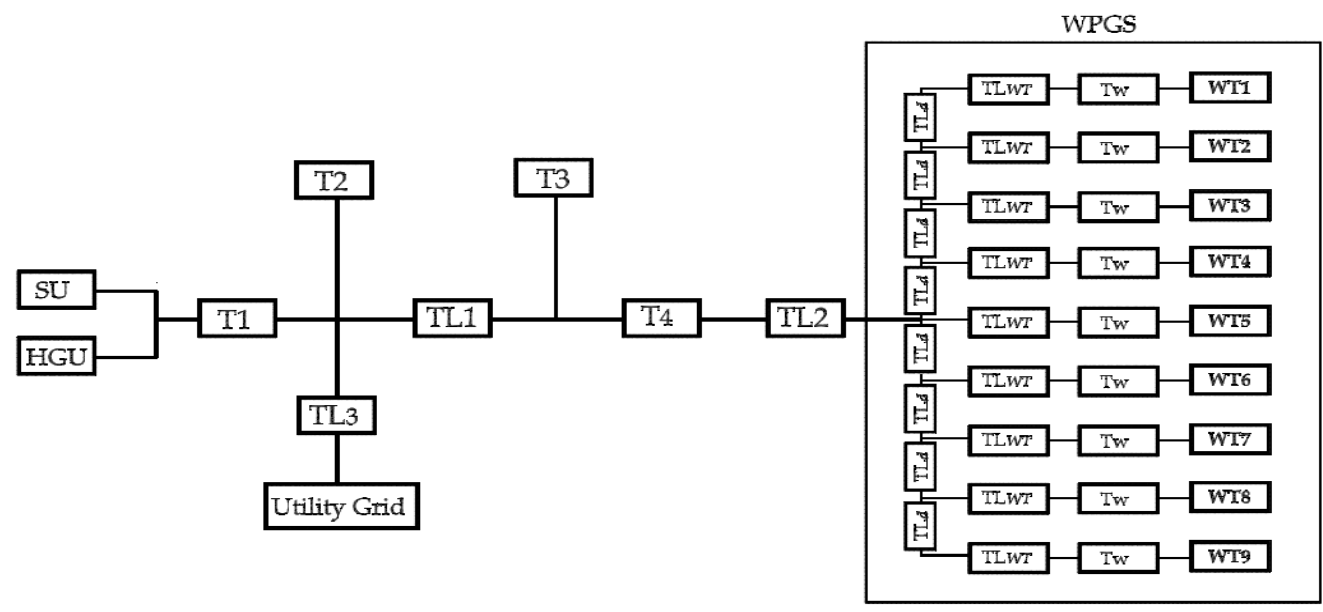

Fig. 3. Detail reliability block diagram of the microgrid system.

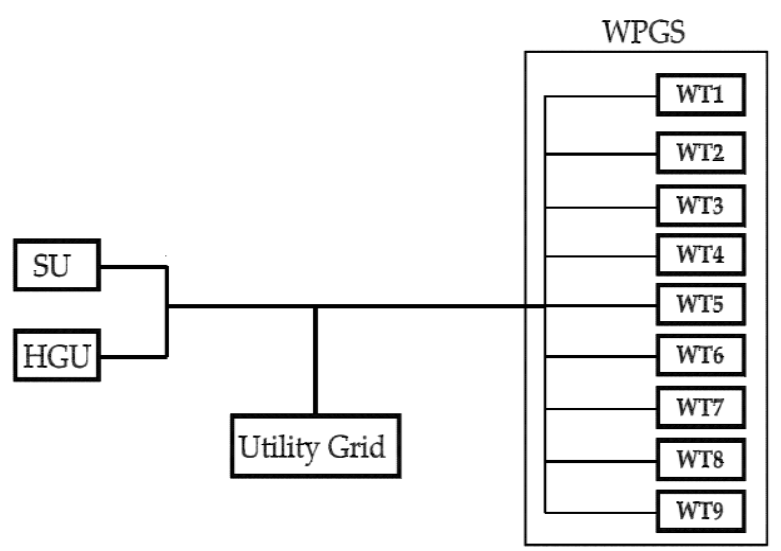

Fig. 4. Simplified reliability block diagram of the microgrid system. 


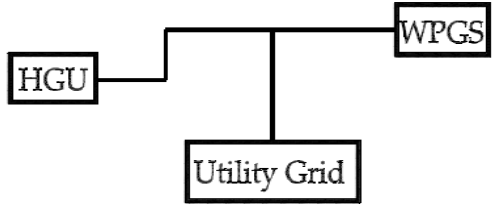

(a)
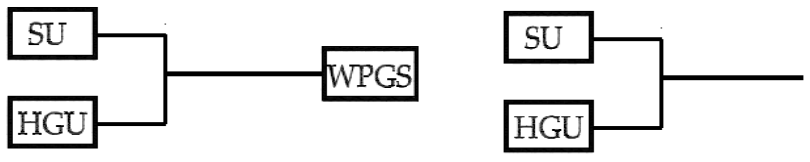

(b)

(c)

Fig. 5. Reliability block diagram: (a) grid connected mode, (b) isolated microgrid with wind power generation system, (c) isolated microgrid without wind power generation system.

Moreover, in order to estimate reliability of a DG unit, the various sub-systems in a DG unit can also be represented by the RBD. The RBD of a wind generator system is shown in Fig. 6, which consists of WT or WT rotor, gearbox, generator and power electronics interfacing circuitry. In this study, HGU and utility grid are assumed highly reliable generation sources. The reliability assessment of a Storage Unit (SU) is beyond the scope this paper, however, the reliability level of the SU is assumed for the sake of reliability calculation of the isolated microgrid system without WPGS (Fig. 5(c)).

\section{Reliability Modelling}

Monte Carlo simulation treats the occurrence of failures as a random event, which mimic the wind speed distribution [30]. For example, in a time series wind data, some of the wind data are not sufficient to produce power, which can be considered as, failure events and the events occur randomly. In addition, this research focuses to assess the reliability of generating and supplying power of the microgrid system while the wind speed is considered as the main uncertainty in the system. Thus, Monte Carlo simulation based reliability assessment for the microgrid system is considered in this paper.

\subsection{Wind speed data modelling}

The relation between wind speed and a WT rotor power output is expressed as [31]

$P_{r o}=0.5 \rho A_{S A} C_{p}(\lambda, \beta) v^{3}$

where, $A_{S A}$ is the swept area covered by the turbine rotor, $C_{p}$ is the power coefficient, $v_{w}$ is the wind velocity, $\beta$ is the pitch angle of rotor blades, $\lambda$ is the tip speed ratio, $\rho$ is the air density. For a given WT, $A_{S A}, C_{p}, \beta, \lambda$, and $\rho$ are constant. The relation in eq. 2 can be expressed as

$P_{r o} \infty v_{w}^{3}$

Since wind speed is the main factor that creates uncertainty at the power output of a WECS system, wind speed is considered here as the key factor to estimate the MSR. In order to relate wind speed effects in reliability estimation, wind speed field data modeling is essential because the data itself varies not only from site to site but also varies according to the hub heights of the wind turbine. Wind speed data modeling for a wind turbine system includes:

a. Identifying best-fit distribution for one-year wind field data

b. Evaluating the goodness-of-fit test

c. Estimating the distribution parameters

a) Identification of best-fit distribution:

Probability plots method is used to identify the best-fit distribution of the available wind data for a given site and for a given wind turbine hub height [29]. The following steps are taken to accomplish the 
fitting of the wind data to a distribution:

- Obtain one-year wind speed data from the site measurement

- Scale the wind data according to the hub height of the wind turbine using eq. 4

$v_{w 2}=v_{w 1}\left(\frac{h_{2}}{h_{1}}\right)^{\alpha}$

where, $h_{1}$ and $h_{2}$ are the height of anemometer and hub, respectively, $v_{w 1}$ and $v_{w 2}$ are the wind velocity at anemometer height and at the hub height, respectively, and $\alpha$ is the shear exponent that is expressed as

$$
\alpha=\left(0.096 \log \left(Z_{o}\right)+0.016 \log \left(Z_{o}\right)\right)^{2}+0.24
$$

where, $Z_{0}$ is the surface roughness.

- Use Matlab Distribution Fitting Tool to obtain probability plot of the scaled wind data

- Fit the probability plot of the scaled wind data for different distributions such as normal, log-normal, exponential and Weibull.

- Identify the distribution corresponding to the best fit of the probability plots.

b) Goodness-of-fit test:

The best-fit distribution of the site wind data is tested for the goodness-of-fit and is performed according to the statistic for MANN'S test given as follows in [29]

$$
M=\frac{k_{1} \sum_{i=k_{1}+1}^{r-1}\left[\left(\ln \left(v_{w_{i+1}}\right)-\ln \left(v_{w_{i}}\right)\right) / M_{i}\right]}{k_{2} \sum_{i=1}^{k_{1}}\left[\left(\ln \left(v_{w_{i+1}}\right)-\ln \left(v_{w_{i}}\right)\right) / M_{i}\right]}
$$

c) Distribution parameters estimation:

In order to determine Weibull distribution parameters, least-squares technique is used because of its accuracy to fit a straight line in a given data points [29]. In this approach, the wind speed field data are transformed to Weibull distribution to fit to a linear regression line as in eq. 7

$y_{i}=a+b x_{i}$

where,

$x_{i}=\ln v_{w i}$

$y_{i}=Z_{i}$

$a=-\beta_{w s} \ln \theta$

$b=\beta_{w s}$

The values of $a$ and $b$ are determined from the least-squares fit using eq. 8 and eq. 9. By knowing the values $a$ and $b$, the Weibull parameters are determined as follows

$\theta_{w s}=\exp \left(-\frac{a}{b}\right)$

$\beta_{w s}=b$ 
where, $\theta_{w s}$ and $\beta_{w s}$ are defined as the scale and shape parameters for wind speed field data.

\subsection{Wind power generation system}

According to the microgrid configuration, all nine WTs in WPGS are connected in parallel, which are shown in the simplified RBD in Fig. 4. In order to estimate the reliability of power generation by the WPGS, single WT system is considered because all of them are identical both in terms of topology and sub-systems context. A WT system comprises of different sub-systems is shown in Fig. 6. The different sub-systems are connected in series because failure of power generation by any sub-systems has to be considered as the WT system failure to generate power. The modeling of reliability estimation of different sub-systems in a WT system is described in the following sub-sections:

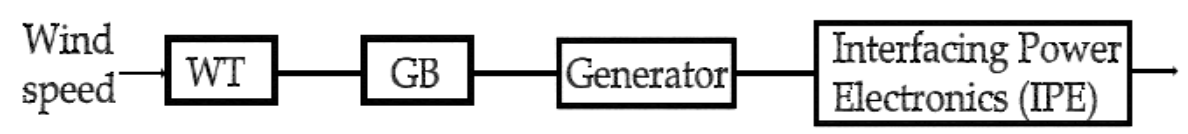

Fig. 6. Reliability block diagram of a wind turbine system.

\section{Wind Turbine Rotor:}

The wind speed field data model provides information about the shape parameter and scale factor for Weibull distribution. Such parameters are used to generate a series of random wind speed data that follows Weibull distribution. Randomly generated data are used to determine power generation by the WT using eq. 2, which represents Weibull distribution of power generation. Weibull parameters for power distribution is determined using parameter estimation technique described in Section 3.1. The Weibull parameters for power distribution are defined as $\theta_{t p}$ and $\beta_{t p}$. So, the reliability of generating power by the WT rotor, $R_{t p}$ can be expressed as

$$
R_{t p}=\exp \left[-\left(\frac{P_{c i w}}{\theta_{t p}}\right)^{\beta_{t p}}\right]-\exp \left[-\left(\frac{P_{c o w}}{\theta_{t p}}\right)^{\beta_{t p}}\right]
$$

where, $\theta_{t p}$ and $\beta_{t p}$ are defined as shape parameter and scale factor for power distribution. $P_{c i w}$ and $P_{c o w}$ are the power at cut-in and cut-out wind speed, respectively.

The reliability of generating power at the $i^{\text {th }}$ wind speed, $R_{P i}$ can be expressed as

$$
R_{P i}=\exp \left[-\left(\frac{P_{i}}{\theta_{t p}}\right)^{\beta_{t p}}\right]
$$

where, $P_{i}$ is the power for $i^{\text {th }}$ wind speed in between cut-in and cut-out region.

\section{Gearbox:}

Weibull parameters obtained from field data modeling is utilized to produce a set of random wind data. Such data are used to determine the wind turbine speed using eq. 16

$$
\omega_{w t}=\frac{\lambda v_{w}}{R_{t}}
$$

where, ${ }^{\omega_{w t}}$ is the wind turbine speed, ${ }^{R}$ is the turbine radius, respectively. The wind turbine speed is also the speed seen by the gearbox low speed shaft. Speed seen by the gearbox can be represented as Weibull distribution of speed. Such distribution is utilized to estimate shape parameter and scale factor for reliability model of gearbox. The reliability of gearbox, ${ }^{R_{g b}}$ can be expressed as 
$R_{g b}=\exp \left[-\left(\frac{\omega_{w t, s}}{\theta_{g b}}\right)^{\beta_{g b}}\right]-\exp \left[-\left(\frac{\omega_{w t, m}}{\theta_{g b}}\right)^{\beta_{g b}}\right]$

where, $\omega_{w t, s}$ is the starting speed of the wind turbine, $\theta_{g b}$ and $\beta_{g b}$ are the shape parameter and scale factor for speed seen by the gearbox, and $\omega_{w t, m}$ is the maximum operating speed of the wind turbine.

The reliability at the $i^{t h}$ speed seen by the gear box, $R_{g b, w t i}$ can be estimated

$$
R_{g b_{w t, i}}=\exp \left[-\left(\frac{\omega_{w t, i}}{\theta_{g b}}\right)^{\beta_{g b}}\right]
$$

where, $\omega_{w t, i}$ is the $i^{t h}$ speed of the WT seen by the gearbox.

\section{Generator:}

In order to account the effect of wind speed in estimating the reliability of generating power by the wind generator, the estimation of Weibull parameters using field data are used. Such parameters are utilized to generate a set of random wind speed data. Power generated by the WT is then determined using eq. 2. However, the power at the generator output depends on the gearbox efficiency and various losses in the generator. Efficiency of the gearbox (0.95) and generator (0.95) is considered as 90 percent, which is observed from the system modeling and simulation. The power at the generator output can be determined as 90 percent of the power at the turbine output. Thus, a power distribution at the generator output can be obtained, which also follows Weibull. Such power distribution at the generator output is used to estimate Weibull distribution parameters using leastsquares parameter estimation technique. After knowing the distribution parameters of the generator output power, the reliability of generating power by the generator, $R_{g}$ can be evaluated as

$$
R_{g}=\exp \left[-\left(\frac{P_{g, c i w}}{\theta_{g p}}\right)^{\beta_{g p}}\right]-\exp \left[-\left(\frac{P_{g, c o w}}{\theta_{g p}}\right)^{\beta_{g p}}\right]
$$

where, $\theta_{g p}$ and $\beta_{g p}$ are considered as shape parameter and scale factor for the generator power distribution. $P_{g, \text { ciw }}$ and $P_{g, \text { cow }}$ are the generator power at the cut-in and cut-out wind speed, respectively.

The reliability of generating power $P_{g, i}$ of the generator, $R_{P_{g, i}}$ can be expressed as

$$
R_{P_{g, i}}=\exp \left[-\left(\frac{P_{g, i}}{\theta_{g p}}\right)^{\beta_{g p}}\right]
$$

where, $P_{g, i}$ is the generator power at the $i^{t h}$ wind speed in between cut-in and cut-out region.

\section{Power Electronics Interfacing System:}

Interfacing Power Electronics (IPE) system in a doubly fed induction generator based WT consists of back-to-back pulse width modulated (PWM) converter as shown in Fig. 7. The components in the IPE system are diodes, IGBT switches and a DC bus capacitor. The reliability model of such a system can be developed based on the relationship between the lifetime and failure rate of the components in the system.

The lifetime of the components are determined considering junction temperature as a co-variate [28]. The junction temperature, $T_{j}$ of a semiconductor device can be calculated as [32].

$$
T_{j}=T_{a}+P_{l} R_{j a}
$$




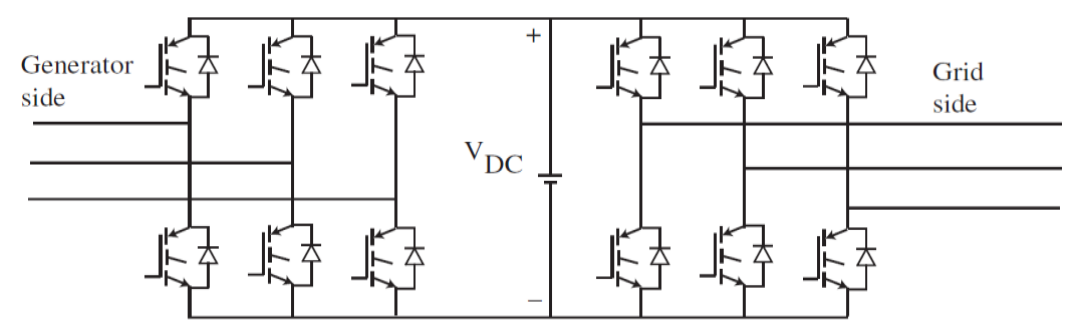

Fig. 7. Interfacing power electronics system of a doubly fed induction generator based wind turbine system.

where, $P_{l}, T_{a}, R_{j a}$ are the power loss of a component, the ambient temperature, and the junction resistance, respectively. Reliability model of a power conditioning system for a small $(1.5 \mathrm{~kW})$ wind energy conversion system is developed by considering power loss only at a rated wind speed operating condition [28]. However, it is to be noted that power losses in the semiconductor components vary according to the wind speed variation at the wind turbine input. Thus, a power loss variation in the semiconductor components is important to consider as a stress factor to calculate the lifetime of the components instead of using power loss quantity for a single operating condition. Hence, the eq. 21 can be expressed as

$T_{j_{i}}=T_{a}+P_{l_{i}} R_{j a}$

where, $\boldsymbol{P}_{l_{i}}$ is the power loss of a component at the $i^{\text {th }}$ wind speed, $T_{j_{i}}$ is the component junction temperature at the $i^{\text {th }}$ wind speed, and junction resistance is assumed to be constant for all wind speed.

In IPE system, there are two types of semiconductor components namely, diode and IGBT switches. Two types of power losses such as conduction losses and switching losses occurs in such components. The conduction loss, $P$ cl, $d$ and switching loss, $P_{s l, d}$ of a diode can be expressed as [33], [34].

$$
\begin{aligned}
P_{c l, d} & =\left(\frac{1}{8}-\frac{M}{3 \pi} \cos \varphi\right) R_{d} I_{m o}^{2}+\left(\frac{1}{2 \pi}-\frac{M}{8} \cos \varphi\right) V_{F O} I_{m o} \\
P_{s l, d} & =\frac{1}{\pi} f_{s} E_{s r} \frac{V_{d c} I_{m o}}{V_{r e f, d} I_{r e f, d}}
\end{aligned}
$$

Total power losses of diodes, $P_{t l, d}$ in the IPE system can be expressed as the sum of the conduction loss, $P c l, d$ for total number of diodes and the switching loss, $P_{s l, d}$ for total number of switches in the system and can be expressed as

$$
P_{t l, d}=n\left(\frac{1}{8}-\frac{M}{3 \pi} \cos \varphi\right) R_{d} I_{m o}^{2}+n\left(\frac{1}{2 \pi}-\frac{M}{8} \cos \varphi\right) V_{F O} I_{m o}+n \frac{1}{\pi} f_{s} E_{s r} \frac{V_{d c} I_{m o}}{V_{r e f, d} I_{r e f, d}}
$$

where, $\mathrm{M}$ is the modulation index $(0 \leq \mathrm{M} \leq 1)$, Imo maximum output current of the inverter, $n$ is the number of semiconductor components, $V_{F O}$ and $R_{d}$ are the diode threshold voltage and resistance, respectively. $f_{s}$ is the switching frequency, $E_{s r}$ is the rated switching loss energy given for the commutation voltage and current $V_{r e f, d}$ and $I r e f, d$. $V d c$ and $I d c$ are the actual commutation voltage and current, respectively and $\varphi$ is the angle between voltage and current.

The conduction loss, $P_{c l, I G B T}$ and switching loss $P_{s l, I G B T}$ of a IGBT switch can be expressed as [33], [35]

$$
P_{c l, I G B T}=\left(\frac{1}{8}+\frac{M}{3 \pi} \cos \varphi\right) R_{c e} I_{m o}^{2}+\left(\frac{1}{2 \pi+\frac{M}{8} \cos \varphi}\right) V_{C E O} I_{m o}
$$


$P_{s l, I G B T}=\frac{1}{\pi} f_{s}\left(E_{o n}+E_{o f f}\right) \frac{V_{d c} I_{m o}}{V_{r e f, I G B T} I_{r e f, I G B T}}$

Total power losses of switches, $P_{t l, I G B T}$ in the IPE system can be expressed as the sum of the conduction loss, $P_{c l, I G B T}$ for total number of diodes and the switching loss, $P_{s l, I G B T}$ for total number of switches in the system, and can be expressed as

$$
P_{t l, I G B T}=n\left(\frac{1}{8}+\frac{M}{3 \pi} \cos \varphi\right) R_{c e} I_{m o}^{2}+n\left(\frac{1}{2 \pi}+\frac{M}{8} \cos \varphi\right) V_{C E O} I_{m o}+n \frac{1}{\pi} f_{s}\left(E_{o n}+E_{o f f}\right) \frac{V_{d c} I_{m o}}{V_{r e f, I G B T} I_{r e f, I G B T}}
$$

where $V_{C E O}$ and $R_{C e}$ are the IGBT threshold voltage and on-state resistance, respectively. Reference commutation voltage and current are $V_{\text {ref,IGBT }}$ and $I_{\text {ref,IGBT }}$, while $V_{d c}$ is the actual commutation voltage. $E_{o n}$ and $E_{\text {off }}$ are the turn-on and turn-off energies of IGBT.

The lifetime, $L(T j i)$ of a component for $i^{\text {th }}$ wind speed can be expressed as

$$
L\left(T_{j i}\right)=L_{o} \exp \left(-B \Delta T_{j i}\right)
$$

where $L_{o}$ is the quantitative normal life measurement (assumed to be $10^{6}$ ). $\mathrm{B}=\frac{E_{A}}{K}$, where $\mathrm{K}$ is the Boltzmann's constant $\left(=8.6 \times 10^{-5} \mathrm{eV} / \mathrm{K}\right), E_{A}$ is the activation energy $(=0.2 \mathrm{eV})$ for typical semiconductor components [29]. $\Delta T j i$ is the variation in junction temperature for the $i^{\text {th }}$ wind speed and can be expressed as

$$
\Delta T_{j i}=\frac{1}{T_{a}}-\frac{1}{T_{j i}}
$$

The failure rate of a component for $i^{\text {th }}$ wind speed can be defined as

$$
\tau_{i}=\frac{1}{L\left(T_{j i}\right)}
$$

Using eq. 31, a distribution of failure rates for a set of wind speed data for a semiconductor component can be generated. The components in the IPE system are considered in series connection from reliability point of view, because the IPE system fails, if any one of components fails in the IPE system. Thus, the failure rates for different components are added to determine the failure rate of the IPE system for $i^{\text {th }}$ wind speed. Hence, a distribution of failure rates for the IPE system can be generated for a series of wind speed data. A least-squares technique is then used to determine the distribution parameters. By knowing the distribution parameters, the reliability of the IPE system, RIPE can be modeled as

$$
R_{I P E}=\exp \left[-\left(\frac{\tau_{c i w}}{\theta_{I P E}}\right)^{\beta_{I P E}}\right]-\exp \left[\left(\frac{\tau_{c o w}}{\theta_{I P E}}\right)^{\beta_{I P E}}\right]
$$

where, $\theta_{I P E}$ and $\beta_{I P E}$ are defined as shape parameter and scale factor for the failure rate distribution of the IPE system. $\tau_{c i w}$ and $\tau_{c o w}$ are failure rates of IPE system at cut-in and cut-out wind speed, respectively.

The reliability of a component in IPE system, RIPEc can be expressed as

$$
R_{I P E_{C}}=\exp \left[-\left(\frac{\tau_{c i w_{C}}}{\theta_{I P E_{C}}}\right)^{\beta_{I P E_{C}}}\right]-\exp \left[\left(\frac{\tau_{c o w_{C}}}{\theta_{I P E_{C}}}\right)^{\beta_{I P E_{C}}}\right]
$$


where, $\theta_{I P E_{C}}$ and $\beta_{I P E_{C}}$ are defined as shape parameter and scale factor for the failure rate distribution of a component. $\tau_{c i w_{C}}$ and $\tau_{c o w_{C}}$ are failure rates at cut-in and cut-out wind speed for a component, respectively.

The reliability of a WT system, $R_{w t s}$ can now be expressed as

$$
R_{w t s}=R_{t p} \times R_{g b} \times R_{g} \times R_{I P E}
$$

In WPGS, all nine WTs are connected in parallel with identical configuration. Hence, the reliability of the WPGS, RWPGS can be expressed as

$$
R_{W P G S}=\left[1-\left(1-R_{w t s}\right)^{N}\right]
$$

where, $\mathrm{N}$ is the number of WT system in a WPGS.

\subsection{Micro-grid reliability model}

Fig. 4 shows the simplified RBD of the microgrid system, where all DG units are connected in parallel. As SU is not always generating power in the microgrid system, it is to be considered in the reliability model in case of generating unit only. Assuming the reliability of the HGU as $R_{H G U}$ and utility grid as $R_{U G}$, the overall microgrid system reliability, $R M S R$ can be modeled as

$$
R_{M S R}=\left[1-\left(1-R_{w t s}\right)^{N}\left(1-R_{H G U}\right)\left(1-R_{U G}\right)\right]
$$

However, the microgrid system operates in three different modes, which are shown in Fig. 5. The MSR can also be modeled according to their operating modes. Fig. 5(a) shows the grid connected mode of operation where all DG or generation units are connected with the utility grid. Thus the MSR for grid connected mode of operation, $R_{M S R M I}$ can be expressed by the similar equation presented in (36). Therefore

$$
R_{M S R_{M 1}}=\left[1-\left(1-R_{w t s}\right)^{N}\left(1-R_{H G U}\right)\left(1-R_{U G}\right)\right]
$$

Fig. 5(b) represents isolated microgrid system with WPGS. In addition, the storage unit is not working as a generation unit in this mode of operation. Thus the MSR during isolated operation with WPGS, $R_{M S R_{M 2}}$ can be defined as

$$
R_{M S R_{M 2}}=\left[1-\left(1-R_{w t s}\right)^{N}\left(1-R_{H G U}\right)\right]
$$

Furthermore, Fig. 5(c) shows isolated microgrid without WPGS mode where the SU operates as a generation unit. Assume that the reliability of the $\mathrm{SU}$ is $R_{S U}$. Hence the MSR during this mode, $R_{M S R_{M 3}}$ can be written as

$$
R_{M S R_{M 3}}=\left[1-\left(1-R_{H G U}\right)\left(1-R_{H G U}\right)\right]
$$

\section{Implementation of the Microgrid Reliability Model}

In order to implement the developed MSR model to evaluate power generation reliability of the proposed microgrid system, Monte Carlo simulation is performed using Matlab. The flow diagram of implementing the reliability model of wind generation system is shown in Fig. 8. The detail of the flow diagram is explained in steps 1-5. The model of the MSR and the reliability evaluation of various 
operating modes of the proposed microgrid are implemented using Matlab code according to the flow chart shown in Fig. 9. The detail of the flow chart is explained in steps 6-7.

STEP 1: Wind speed field data model

- Field data collection and distribution identification using probability plots

- Goodness-of-fit test for selecting the distribution of wind speed

- Calculate the distribution parameter using eq. 12 and 13

- Generate a series of random data as the input for next steps of the reliability flow diagram

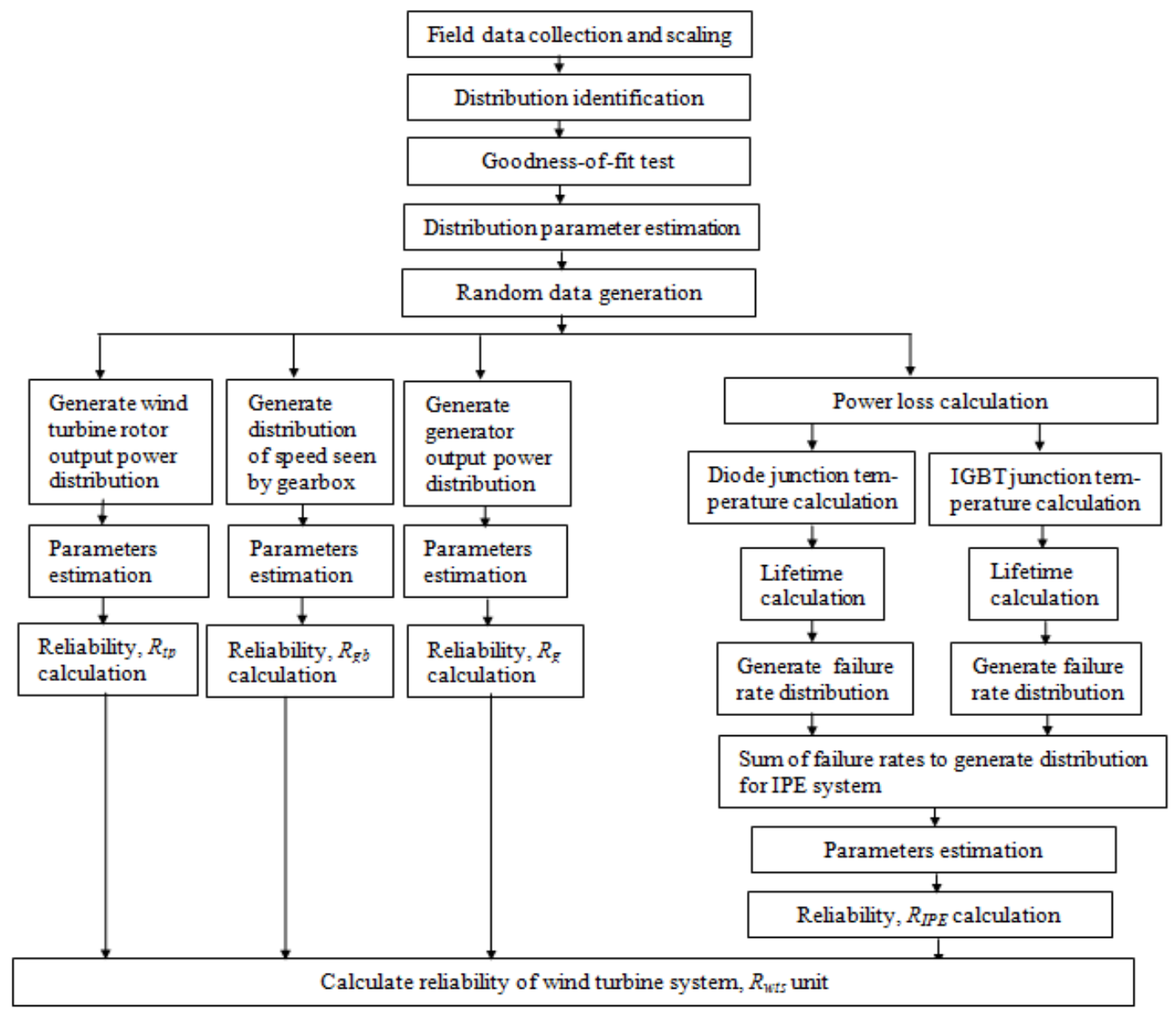

Fig. 8. Flow diagram for reliability calculation of wind generation sub-systems.

STEP 2: Reliability of power generation by WT rotor

- WT rotor output power distribution generation

- Parameter estimation for WT rotor power distribution

- Reliability calculation using eq. 14

STEP 3: Reliability of gearbox

- Determine speed distribution seen by the gearbox

- Speed distribution parameter calculation using least-squares technique

- Reliability calculation using eq. 17

STEP 4: Reliability of generator 
- Generator output power distribution generation

- Distribution parameter determination using least-squares technique

- Reliability evaluation of generator output power using eq. 19

STEP 5: Reliability of interfacing power electronics

- Power loss calculation of diodes and IGBTs in the IPE system using eq. 25 and 28

- Failure rate distributions generation for diodes and IGBT switches

- Estimate parameter of failure rate distribution of IPE system

- Calculate reliability using eq. 32

STEP 6: Reliability of DG units

- Reliability calculation of a WT system using eq. 34

- Determine reliability of WPGS using eq. 35

- Assume reliability for HGU and SU

STEP 7: Reliability of micro-grid system

- MSR calculation using eq. 36, 37, 38 and 39 for various operational modes

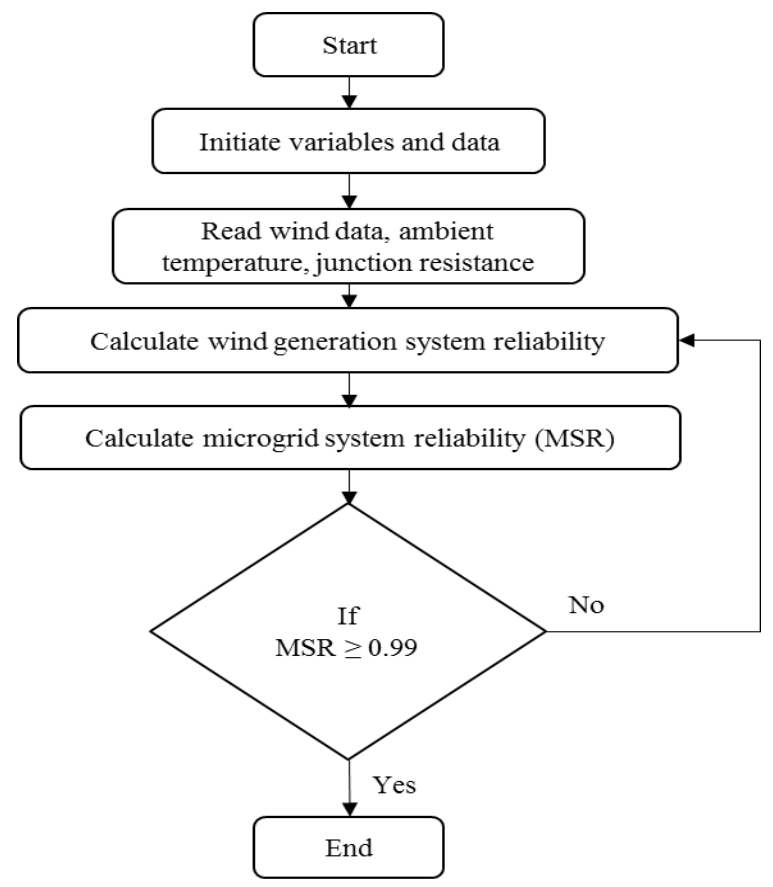

Fig. 9. Flow chart for calculating the microgrid system reliability.

\section{Simulation Results}

The reliability model and its implementation procedure described in the preceding sections are performed to determine probability distribution parameters as well as the reliability of the various subsystems in the wind generation system for stochastically varying wind speed condition. Such reliability estimation is then utilized to determine MSR in various operating modes of the microgrid. The power generation wind speed region of the selected turbine is $v_{c i w}=4 \mathrm{~m} / \mathrm{s}$ and $v_{\text {cow }}=25 \mathrm{~m} / \mathrm{s}$. The reliability of HGU and utility grid are assumed as 85 percent, since they are assumed as highly reliable power generation sources. The reliability of storage unit is assumed to be same as the IPE system $(=0.8144)$, because the storage units are commonly interfaced through power electronics inverter system. The IGBT module and diode parameters for loss calculation are obtained from [28]. One-year wind 
speed data is used for the field data modeling process. Assume that three WT systems can be connected to the isolated microgrid system due to the stability issue.

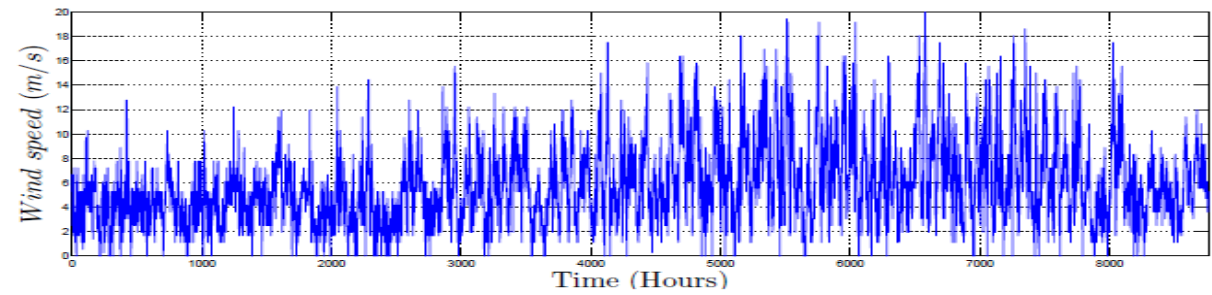

Fig. 10. Wind speed field data.

Fig. 10 shows the hourly wind speed field data collected over one-year period. Such data is utilized to identify the distribution using probability plot technique. The probability plots of wind speed field data are shown in Fig. 11. It can be seen from Fig. 11 that the probability of wind speed follows Weibull and Rayleigh distributions closely; however, the Weibull distribution follows the probability of wind speed closer than the Rayleigh distribution. Thus, the Weibull distribution is identified as the best-fit distribution for wind speed data in this study. In order to select Weibull distribution, a goodness-of-fit test is also carried out and the probability density function of Weibull distribution is shown in Fig. 12.

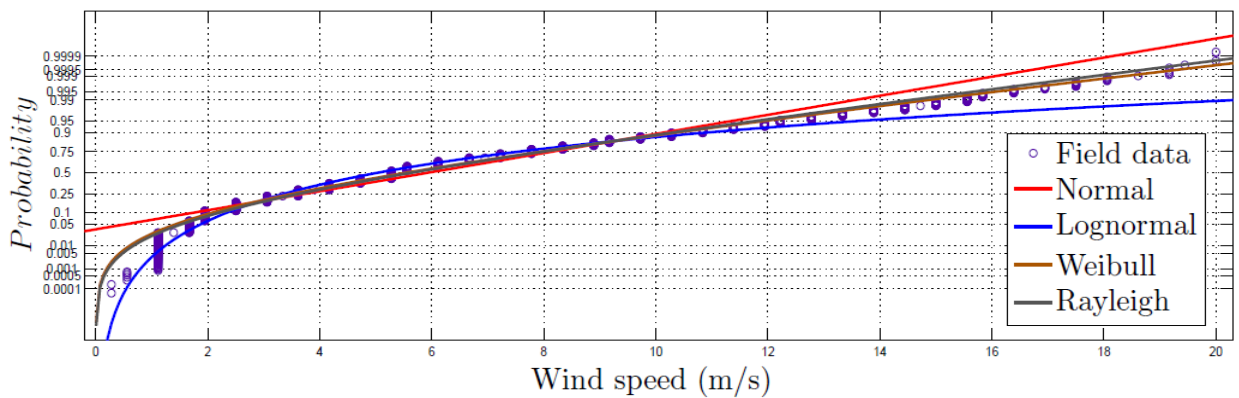

Fig. 11. Probability plots for distribution identification.

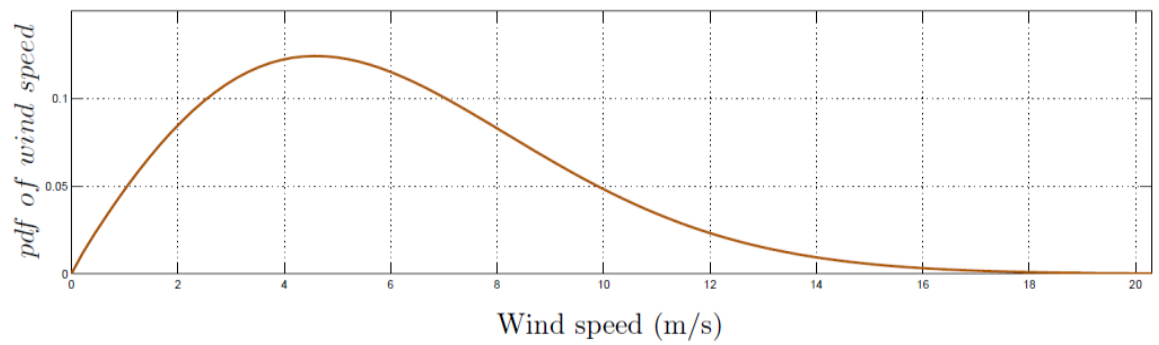

Fig. 12. Probability density function of wind speed data.

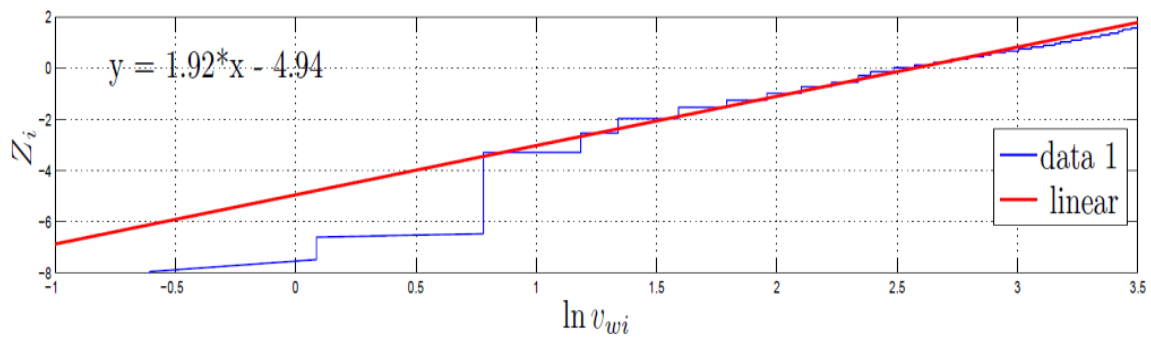

Fig. 13. Least-squares plot for parameters estimation.

A least-squares method is followed to estimate the Weibull distribution parameter, which is shown in 
Fig. 13. The shape parameter for wind speed, $\beta_{w s}=1.92$ and the scale parameter, $\theta_{w s}=13.1$. These parameters are used to generate random wind speed data for reliability evaluation of different sub-systems in a wind turbine system.

The results of reliability calculation for different subsystems in a wind generation system are presented in Table 1. The results reveal that the reliability of generating power by wind turbine rotor is 0.9068 , while the reliability of gearbox and generator are 0.9107 and 0.9266 , respectively. However, the reliability of generating power for IPE sub-system is only 0.8144 . These results indicate that the IPE subsystem in a variable speed wind generator system is less reliable than the other sub-systems. Table 2 presents the reliability results of DG units such as WT system, WPGS, HGU, SU and utility grid. The reliability of a WT system and a WPGS is calculated based on the model derived in this study, however, the reliability of HGU, SU and utility grid are assumed. The overall reliability of a wind turbine system is 0.6232. Since nine WT systems are connected in parallel in the WPGS, the calculated reliability of WPGS is significantly high.

Table 1. Reliability results of different sub systems in a variable speed wind generator system

\begin{tabular}{|c|c|c|c|c|c|}
\hline \multirow{3}{*}{$\begin{array}{l}\text { Sub-systems } \\
\text { WT rotor }\end{array}$} & \multicolumn{2}{|c|}{ Distribution parameters } & \multicolumn{2}{|c|}{ Sub-systems parameters } & \multirow{2}{*}{$\begin{array}{c}\text { Reliability } \\
R_{t p}\end{array}$} \\
\hline & $\theta_{t p}$ & $\beta_{t p}$ & $P_{c i w}$ & $P_{\text {cow }}$ & \\
\hline & 1560.58 & 1.422 & 77 & 3000 & 0.9068 \\
\hline \multirow{2}{*}{ Gearbox } & $\theta_{g b}$ & $\beta_{g b}$ & $\omega_{w t, s}$ & $\omega_{w t, \mathrm{~m}}$ & $R_{g b}$ \\
\hline & 13.73 & 3.33 & 4.1 & 18.4 & 0.9107 \\
\hline \multirow{2}{*}{ Generator } & $\theta_{g}$ & $\beta_{g}$ & $P_{g, c i w}$ & $P_{g, \text { cow }}$ & $R_{g}$ \\
\hline & 1354 & 1.4142 & 73 & 2850 & 0.9266 \\
\hline \multirow{2}{*}{ IPE system } & $\theta_{I P E}$ & $\beta_{I P E}$ & $\tau_{c i w}$ & $\tau_{\text {cow }}$ & $R_{I P E}$ \\
\hline & 1.158 & $2.658 \mathrm{e}-5$ & $0.0202 \mathrm{e}-4$ & $0.4821 \mathrm{e}-4$ & 0.8144 \\
\hline
\end{tabular}

Table 2. Reliability results of distributed generation units

\begin{tabular}{cc|cc}
\hline DG Units & Reliability & DG Units & Reliability \\
\hline \multirow{2}{*}{ WT system } & $R_{w t s}$ & HGU & $R_{H G U}$ \\
\cline { 2 - 3 } & 0.6232 & & 0.85 \\
\cline { 2 - 3 } WPGS & $R_{W P G S}$ & SU & $R_{S U}$ \\
\cline { 2 - 3 } & 0.9998 & & 0.8144 \\
\hline
\end{tabular}

Table 3. Reliability results of microgrid system

\begin{tabular}{lc}
\hline \multicolumn{1}{c}{ Microgrid operational modes } & Reliability \\
\hline Grid connected mode & $R_{M S R_{M 1}}$ \\
\cline { 2 - 2 } & 0.9999 \\
\hline Isolated microgrid with WPGS: number of WTs in WPGS $(1,2,3,4)$ & $R_{M S R_{M 2}}$ \\
\hline Isolated microgrid without WPGS & $0.94,0.97,0.99,0.997$ \\
\hline
\end{tabular}

Reliability estimation results of the microgrid system during various operational modes are presented in Table 3. The MSR during grid connected mode is higher than the other operational modes because during this mode all DG units are operating. Moreover, this mode has two generation sources which are assumed as highly reliable in power generation and supply. On the other hand, MSR during isolated 
microgrid with WPGS varies depending on the number of WT system operating in the WPGS. It is worth mentioning that in an isolated microgrid system, all WT system in WPGS does not operate because of the stability issue. For example, all WT systems in WPGS are consumed reactive power from the utility grid during grid connected mode, however, in an isolated mode there is no such reactive power generation source to provide sufficient reactive power for all nine WT systems. Thus, the reliability calculation is carried out for different number of WT systems in the WPGS and the various reliability indexes are found. However, it is important to note that the minimum reliability index is found 0.94 , which is a very good number. Moreover, the reliability level during this mode of operation (Fig. 5(b)) can also be increased by adding more number of generation sources within the maximum number of constraint (maximum number of WT system). The reliability of microgrid system without WPGS is calculated as 0.97 , which is higher than that of microgrid system with WPGS. It is because of the combination of generation sources in this mode of operation (Fig. 5(c)) are highly reliable than the generation source (such as WT) in the WPGS. The results of the reliability evaluation show that the proposed microgrid system has the significant ability to generate sufficient power to ensure the reliable power supply in all operating modes. Such reliability indexes are trustworthy that a microgrid system consists of renewable energy sources such as wind-hydro-storage can generate and supply reliable power.

\section{Conclusions}

Reliability evaluation of generating and supplying reliable power by a microgrid system comprising of variable speed wind generator units is investigated in this paper. This investigation is carried out on a case study microgrid system located at Fermeuse, Newfoundland, Canada. The mathematical model of microgrid system reliability is developed based on Reliability Block Diagram (RBD) concept. In addition, the reliability model of various sub-systems in a variable-speed wind generator unit is developed considering the impact of stochastically varying wind speed. The developed microgrid system reliability model is implemented through Monte Carlo simulation using Matlab coding. The reliability results obtained through simulation are presented and discussed. The reliability performance of generating and supplying reliable power by the case study microgrid system during its various operational modes are found 0.99 (grid-connected mode), 0.99 (isolated microgrid with WPGS), 0.99 (isolated microgrid without WPGS). This implies that the case study microgrid has the ability to generate and supply power to the loads in microgrid domain with high reliability. Such reliability is achieved due to maximizing the use of renewable power that is coming from wind generation system as well as storage unit. In addition, this reliability evaluation approach can be applied to assess reliability of the microgrid system containing other intermittent energy sources such as solar.

\section{Acknowledgement}

This work is supported by a research grant from the National Science and Engineering Research Council (NSERC) of Canada, the Atlantic Innovation Fund (AIF) Canada, and Memorial University of Newfoundland. The author also would like to acknowledge the utility company, Newfoundland Power, Canada for providing the system information and data.

\section{References}

[1] Lasseter RH. Microgrids. In: Proc. of IEEE Power Engineering Society Winter Meeting, 2002:1-4.

[2] Barnes M, Dimeas A, et al. Microgrid laboratory facilities. Presented at: 2005 IEEE International Conference on Future Power System.

[3] Lopes JAP, Madureira AG, Moreira CCLM. A view of microgrids. WIREs Energy Environ, 2013; 2(1):86-103.

[4] Hatziargyriou N, Asano H, Iravani MR, Marnay C. Microgrids-an overview of ongoing research, development and demonstration projects. IEEE Power and Energy Magazine, 2007; 78-94.

[5] Morozumi S. Overview of microgrid research and development activities in Japan. Presented at: 2006 IEEE Symposium on Micro-grids.

[6] Katiraei F, Iravani MR, Lehn PW. Small signal dynamic model of a microgrid including conventional and electronically 
interfaced distributed resources. IET Gener. Transm. Distrib., 2007; 1(3):369-378.

[7] Katiraei F, Abbey C, Bahry R. Analysis of voltage regulation problem for $25 \mathrm{kV}$ distribution network with distributed generation. In: Proc. of IEEE Power Engineering Society General Meeting, 2006:1-8.

[8] Ibrahim H, Ghandour M, Dimitrova M, Ilinca A, Perron J. Integration of wind energy into electricity systems: technical challenges and actual solutions. Energy Procedia, 2011; 6(6):815-824.

[9] Shahabi M, Haghifam MR, Mohamadian M, Nabavi-Niaki SA. Microgrid dynamic performance improvement using a doubly fed induction wind generator. IEEE Trans. on Energy Conversion, 2009; 24(1):137-145.

[10] Majumder R, Ghosh A, Ledwich G, Zare F. Load sharing and power quality enhanced operation of a distributed micro-grid. IET Renew. Power Gener., 2009; 3(2):109-119.

[11] Nayar C. Innovative remote microgrid systems. International Journal of Environment and Sustainability, 2012; 1(3):53-65.

[12] Kawasaki K. Matsumura S, Iwabu K, Fujimuram N, Iima T. Autonomous dispersed control system for independent microgrid. Journal of Electrical Engineering, Japan, 2009; 166(1):1121- 1127.

[13] Li X, Song Y, Han S. Frequency control in microgrid power system combined with electrolyzer system and fuzzy PI controller. Journal of Power Sources, 2008; 180:468-475.

[14] Basu AK, Chaowdhury SP, Chaowdhury S, Ray D, Crossley PA. Reliability study of a microgrid system. IEEE Trans. on Power Systems, 2006; 21(4):1821-1831.

[15] Karki R, Hu P, Billinton R. Reliability evaluation considering wind and hydropower coordination. IEEE Trans. on Power Systems, 2010; 25(2):685-603.

[16] Tanrioven M. Reliability and cost-benefits of adding alternate power sources to an independent micro-grid community. Journal of Power Sources, 2005; 150:136-149.

[17] Zhou P, Jin RY, Fan LW. Reliability and economic evaluation of power system with renewables: a review. Renewable and Sustainable Energy Reviews, 2016; 58:537-547.

[18] Acuna L, Padilla RV, Mercado AS. Measuring reliability of hybrid photovoltaic-wind energy systems: a new indicator. Renewable Energy, 2017; 106:68-77.

[19] Adefarati T, Bansal RC. Reliability assessment of distribution system with the integration of renewable distributed generation. Applied Energy, 2017; 185:158-171.

[20] Li J, Wei W. Probabilistic evaluation of available power of a renewable generation system consisting of wind turbine and storage batteries: a Markov chain method. Journal of Renewable and Sustainable Energy, 2014; 6(1):1493-1501.

[21] Liu X, Chowdhury AA, Koval DO. Reliability evaluation of a wind-diesel-battery hybrid power system. Presented at: 2008 IEEE Industrial and Commercial Power Systems Technical Conference.

[22] Wang L, Singh C. Adequacy assessment of power-generating systems including wind power integration based on ant colony system algorithm. Presented at: 2007 IEEE Lausanne Power Tech.

[23] Bhuiyan FA, Yazdani A. Reliability assessment of a wind power system with integrated energy storage. IET Renewable Power Generation, 2010; 4(3):211-220.

[24] Choi J, Park J, et al.. Probabilistic reliability evaluation of composite power systems including wind turbine generators. In: Proc. of IEEE International Conference on Probabilistic Method Applied to Power Systems, 2010:802-807.

[25] Vallee F, Lobry J, Deblecker O. System reliability assessment method for wind power integration. IEEE Trans. on Power Systems, 2008; 23(3):1288-1297.

[26] Silva AMLD, Manso LAF. Application of Monte-Carlo simulation to generating system Well-being analysis considering renewable sources. European Transactions on Electrical Power, 2007; 17:387-400.

[27] Wen J, Zheng Y, Donghan F. A review on reliability assessment for wind power. Renewable and Sustainable Energy Reviews, 2009; 13:2485-2494.

[28] Arifujjaman M. Performance and reliability comparison of grid connected small wind turbine systems. PhD dissertation. Department of Electrical and Computer Engineering, Memorial University of Newfoundland, St. John's, NL, CA; 2010.

[29] Ebeling CE. An Introduction to Reliability and Maintainability Engineering. Waveland Press; 2010.

[30] Vittal S. Teboul M. Performance and reliability analysis of wind turbines using Monte-Carlo methods based on system transport theory. In: Proc. of Structural Dynamics and Materials Conference, 2005:1-8.

[31] Oettinger FF, Blackburn DL, Rubin S. Thermal characteristics of power transistors. IEEE Trans. on Reliability, 1976; 23(8):831-838.

[32] Ali MH. Wind Energy Systems: Solutions for Power Quality and Stabilization. CRC Press; 2012.

[33] Feix G, Dieckerhoff S, Allmeling J, Schonberger J, Simple methods to calculate IGBT and diode conduction and switching losses. In: Proc. of 13th European Conference on Power Electronics and Applications, 2009:8-10.

[34] Bierhoff MH, Fuchs FW. Semiconductor losses in voltage source and current source IGBT converters based on analytical derivation. In: Proc. of IEEE Power Electrics Specialist Conference, 2004:2836-2842.

[35] Mestha LK, Evans PD. Analysis of on-state losses in PWM inverter. IEE Proceedings, 1989; 136(4):1989. 